\title{
Imidazole: Having Versatile Biological Activities
}

\author{
Amita Verma, Sunil Joshi, and Deepika Singh \\ Department of Pharmaceutical Sciences, Faculty of Health Sciences, Sam Higginbottom Institute of Agriculture, \\ Technology and Sciences (Deemed to be University), Allahabad, Uttar Pradesh 211007, India \\ Correspondence should be addressed to Amita Verma; amitaverma.dr@gmail.com
}

Received 30 May 2013; Revised 1 September 2013; Accepted 3 September 2013

Academic Editor: Qing Li

Copyright (C) 2013 Amita Verma et al. This is an open access article distributed under the Creative Commons Attribution License, which permits unrestricted use, distribution, and reproduction in any medium, provided the original work is properly cited.

Imidazoles have occupied a unique position in heterocyclic chemistry, and its derivatives have attracted considerable interests in recent years for their versatile properties in chemistry and pharmacology. Imidazole is nitrogen-containing heterocyclic ring which possesses biological and pharmaceutical importance. Thus, imidazole compounds have been an interesting source for researchers for more than a century. The imidazole ring is a constituent of several important natural products, including purine, histamine, histidine, and nucleic acid. Being a polar and ionisable aromatic compound, it improves pharmacokinetic characteristics of lead molecules and thus is used as a remedy to optimize solubility and bioavailability parameters of proposed poorly soluble lead molecules. There are several methods used for the synthesis of imidazole-containing compounds, and also their various structure reactions offer enormous scope in the field of medicinal chemistry. The imidazole derivatives possess extensive spectrum of biological activities such as antibacterial, anticancer, antitubercular, antifungal, analgesic, and anti-HIV activities. This paper aims to review the biological activities of imidazole during the past years.

\section{Introduction}

Imidazole nucleus forms the main structure of some wellknown components of human organisms, that is, the amino acid histidine, Vit-B12, a component of DNA base structure and purines, histamine, and biotin. It is also present in the structure of many natural or synthetic drug molecules, that is, cimetidine, azomycin, and metronidazole. Imidazolecontaining drugs have a broaden scope in remedying various dispositions in clinical medicine [1]. Imidazole was first synthesized by Heinrich Debus in 1858, but various imidazole derivatives had been discovered as early as the 1840s. His synthesis used glyoxal and formaldehyde in ammonia to form imidazole [2]. This synthesis, while producing relatively low yields, is still used for creating C-substituted imidazoles (see Scheme 1).

Imidazole is a 5-membered planar ring, which is soluble in water and other polar solvents. It exists in two equivalent tautomeric forms because the hydrogen atom can be located on either of the two nitrogen atoms. Imidazole is a highly polar compound, as evidenced by a calculated dipole of 3.61D, and is entirely soluble in water. Imidazole is amphoteric; that is, it can function as both an acid and a base. The compound is classified as aromatic due to the presence of a sextet of $\pi$ electrons, consisting of a pair of electrons from the protonated nitrogen atom and one from each of the remaining four atoms of the ring.

Some resonance structures of imidazole are shown in Scheme 2.

Medicinal chemistry concerns with the discovery, development, interpretation, and identification of the mechanism of action of biologically active compounds at the molecular level [3].

\section{Pharmacological Activities}

Imidazoles are well-known heterocyclic compounds which are common and have an important feature of a variety of medicinal agents. On the basis of various literature surveys, imidazole derivatives show various pharmacological activities:
(i) antibacterial activity;
(ii) anticancer activity; 
<smiles>[R]C(=O)C([R])=O</smiles>

Scheme 1<smiles></smiles>

SCHEME 2<smiles>COC(=O)/C=C1/C(=O)NC(=S)N1/N=C/c1c[nH]nc1[Al]</smiles>

$1 a-d$

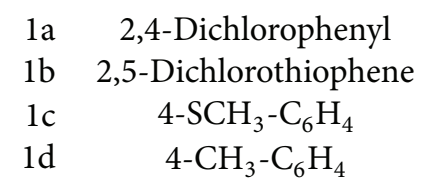<smiles>[X]c1ccc(-c2nc(-c3c[nH]nc3[Al])[nH]c2-c2ccc([X])cc2)cc1</smiles>

$2 \mathrm{a}-\mathrm{j}$

Ar

$\mathrm{X}$

\begin{tabular}{|c|c|}
\hline $2 \mathrm{a}$ & $4-\mathrm{SCH}_{3}-\mathrm{C}_{6} \mathrm{H}_{4}$ \\
\hline $2 b$ & 2,4-Dichlorophenyl \\
\hline . & Biphenyl \\
\hline & $4-\mathrm{CH}_{3}-\mathrm{C}_{6} \mathrm{H}_{4}$ \\
\hline $2 \mathrm{e}$ & 2,5-Dichlorothiophene \\
\hline 21 & $4-\mathrm{SCH}_{3}-\mathrm{C}_{6} \mathrm{H}_{4}$ \\
\hline$g$ & 2,4-Dichlorophenyl \\
\hline & Biphenyl \\
\hline & $4-\mathrm{CH}_{3}-\mathrm{C}_{6} \mathrm{H}_{4}$ \\
\hline & 2,5-Dichlorothiophene \\
\hline
\end{tabular}

SCHEMe 3 (iii) antitubercular activity;

(iv) antifungal;

(v) analgesic activity;

(vi) anti-HIV activity.

\section{Antibacterial Activity}

Vijesh et al. carried out the in vitro antibacterial activity of newly synthesized compounds 1a-d and 2a-j. Escherichia coli, Staphylococcus aureus, Bacillus subtilis, Salmonella typhimvrium, Clostridium perfringens, and Pseudomonas aeruginosa were used to investigate the activity. The antibacterial screening revealed that some of the tested compounds showed good inhibition against various tested microbial strains. 1c showed excellent activity against $P$. aeruginosa and C. perfringens compared to standard drug streptomycin [4] (see Scheme 3).

A series of substituted 4-(2,6-dichlorobenzyloxy)phenyl thiazole, oxazole, and imidazole derivatives (3a-f, $4 a-e)$ were synthesized by $\mathrm{Lu}$ et al. The derivatives were screened for in vitro antibacterial activity against $S$. aureus, E. coli, S. pneumonia, and penicillin-resistant S. pneumonia [5] (see Scheme 4). 
<smiles>[Z15][Z15][H]</smiles>

$\begin{array}{lll} & \mathrm{R} & \mathrm{R}_{1} \\ \text { 3a } & \mathrm{Et} & \mathrm{Ph} \\ \text { 4a } & \mathrm{H} & \mathrm{Ph} \\ 3 \mathrm{~b} & \mathrm{Et} & 4-\mathrm{Cl}-\mathrm{Ph} \\ \text { 4b } & \mathrm{H} & 4-\mathrm{Cl}-\mathrm{Ph} \\ 3 \mathrm{c} & \mathrm{Et} & 2,4-\mathrm{Cl}-\mathrm{Ph} \\ \text { 4c } & \mathrm{H} & 2,4-\mathrm{Cl}-\mathrm{Ph} \\ \text { 3d } & \mathrm{Et} & 4-\mathrm{CH}_{3} \mathrm{O}-\mathrm{Ph} \\ \text { 4d } & \mathrm{H} & 4-\mathrm{CH}_{3} \mathrm{O}-\mathrm{Ph} \\ 3 \mathrm{e} & \mathrm{Et} & n-\mathrm{Pr} \\ \text { 4e } & \mathrm{H} & n-\mathrm{Pr} \\ \text { 3f } & \mathrm{Et} & \text { Ethylene }\end{array}$

SCHEME 4

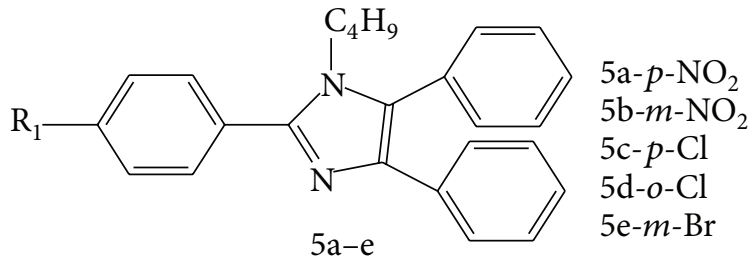

SCHEME 5<smiles>[R]c1ccc(C2C([R])C(=O)C([R])C(c3ccc([R])c([R])c3)N2C(=O)Cn2ccnc2)cc1[R]</smiles>

$6-11$<smiles>[R]c1ccc(C2C([R])C(=O)C([R3])C(c3ccc([R])c([R])c3)N2C(=O)Cn2nnc3ccccc32)cc1[R]</smiles>

$12-15$

$\begin{array}{ccccc}\text { Compounds } & \mathrm{R} & \mathrm{R}_{1} & \mathrm{R}_{2} & \mathrm{R}_{3} \\ 6 & \mathrm{H} & \mathrm{H} & \mathrm{CH}_{3} & \mathrm{H} \\ 7 & \mathrm{H} & \mathrm{H} & \mathrm{CH}_{3} & \mathrm{H} \\ 8 & \mathrm{H} & \mathrm{H} & \mathrm{CH}\left(\mathrm{CH}_{3}\right)_{2} & \mathrm{H} \\ 9 & \mathrm{H} & \mathrm{H} & \mathrm{CH}_{3} & \mathrm{CH}_{3} \\ 10 & \mathrm{~F} & \mathrm{H} & \mathrm{CH}_{3} & \mathrm{CH}_{3} \\ 11 & \mathrm{H} & \mathrm{OCH}_{3} & \mathrm{CH}_{3} & \mathrm{CH}_{3} \\ 12 & \mathrm{~F} & \mathrm{H} & \mathrm{CH}_{3} & \mathrm{CH}_{3} \\ 13 & \mathrm{H} & \mathrm{F} & \mathrm{CH}_{3} & \mathrm{CH}_{3} \\ 14 & \mathrm{H} & \mathrm{OH}_{3} & \mathrm{CH}_{3} & \mathrm{CH}_{3} \\ 15 & \mathrm{H} & \mathrm{F} & \mathrm{CH}_{2} \mathrm{CH}_{3} & \mathrm{H}^{2}\end{array}$

SCHEME 6

Jain et al. synthesize 2-substituted-4,5-diphenyl-N-alkyl imidazole derivatives. and evaluate their antibacterial activity. All the synthesized compounds were evaluated for antibacterial activity against $S$. aureus, B. subtilis, and E. coli. Out of $5 \mathrm{a}-\mathrm{e}$ only $5 \mathrm{a}$ and $5 \mathrm{~b}$ showed some short of activity but none of them had considerable activity compared with that of the standard [6] (see Scheme 5).
Ramachandran et al. synthesized imidazole/benzotriazole substituted piperidin-4-one derivatives. Compounds 6-15 were screened for their in vitro antibacterial activity against Staphylococcus aureus, Bacillus subtilis, Salmonella typhi, Escherichia coli, and Klebsiella pneumonia. Among the compounds, 7 and 10 against $B$. subtilis, 9 against $S$. aureus, 8 and 13 against K. pneumonia, and 15 against $E$. coli did not 


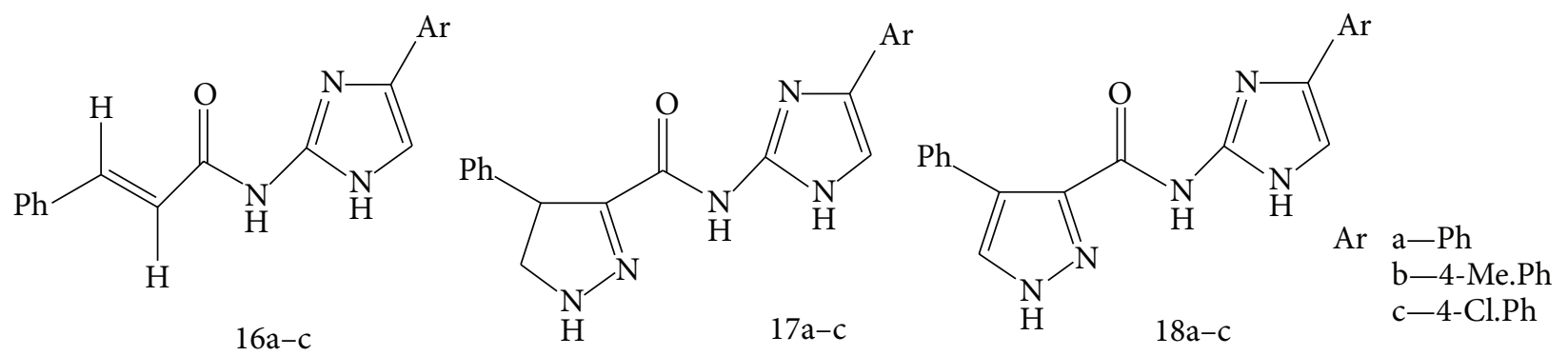

SCheme 7

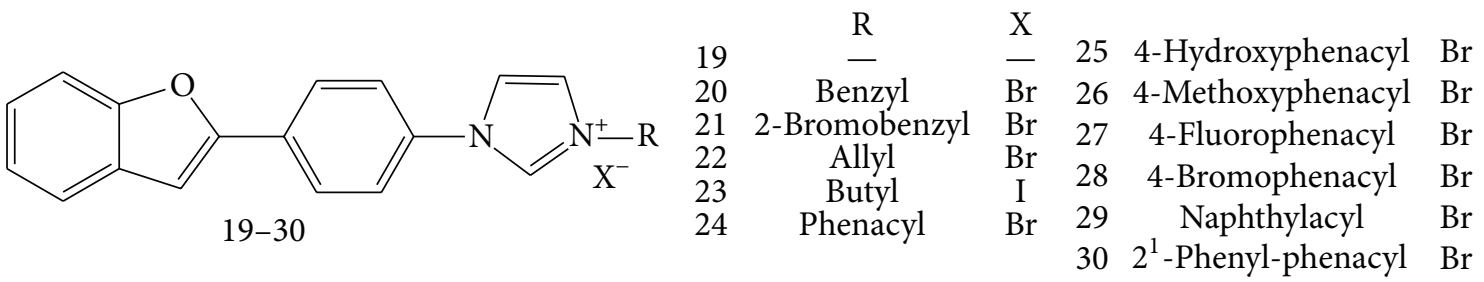

Scheme 8<smiles>CCCCCn1c(C)nc2cc(Cl)c(Cl)cc21</smiles>

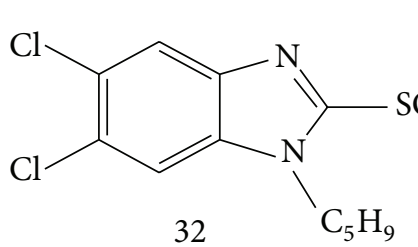<smiles>CCCCCn1c(S(=O)(=O)OC)nc2cc(Cl)c(Cl)cc21</smiles>

SCheme 9<smiles>Cn1c(-c2ccc(NC(=O)CCl)cc2)nc(-c2ccccc2)c1-c1ccccc1</smiles><smiles>[R]SCC(=O)Nc1ccc(-c2nc(-c3ccccc3)c(-c3ccccc3)n2C)cc1</smiles><smiles>[R15]CCCCNc1ccc(-c2nc(-c3ccccc3)c(-c3ccccc3)n2C)cc1</smiles>

$\begin{array}{ll}35=\mathrm{C}_{7} \mathrm{H}_{5} \mathrm{NO} & 41=\mathrm{C}_{3} \mathrm{H}_{4} \mathrm{~N}_{2} \mathrm{~S} \\ 36=\mathrm{C}_{7} \mathrm{H}_{4} \mathrm{ClNO} & 42=\mathrm{C}_{3} \mathrm{H}_{5} \mathrm{NS} \\ 37=\mathrm{C}_{8} \mathrm{H}_{7} \mathrm{NO} & 43=\mathrm{C}_{4} \mathrm{H}_{6} \mathrm{~N}_{2} \\ 38=\mathrm{C}_{7} \mathrm{H}_{5} \mathrm{NS} & 44=\mathrm{C}_{6} \mathrm{H}_{5} \\ 39=\mathrm{C}_{3} \mathrm{H}_{5} \mathrm{~N}_{3} & 45=\mathrm{C}_{6} \mathrm{H}_{5} \mathrm{Cl} \\ 40=\mathrm{C}_{2} \mathrm{H}_{4} \mathrm{~N}_{4} & \end{array}$

SCHeme 10

show any inhibitory activity even at maximum concentration. However, piperidine ring containing compounds 8 against $B$. subtilis and 9 against $E$. coli explored good inhibitory activity. Compound 13 increased the growth inhibition activity against E. coli. And compound 15 showed superior inhibition activity against $B$. subtilis [7] (see Scheme 6).
Padmavathi et al. synthesized amido linked imidazoles derivatives and screened antibacterial activity; it was seen that $16 \mathrm{c}$ and $18 \mathrm{c}$ were more effective against Pseudomonas aeruginosa. Amongst bis heterocyclic compounds, the aromatized bis heterocycle 18 was effective than the corresponding nonaromatized compound 17 . The compounds $16 \mathrm{c}$ and $18 \mathrm{c}$ 
<smiles>[R][X]c1cncn1C(c1ccccc1)c1cc2ccccc2o1</smiles>

Imidazole ring 46-imidazole 47-2-methyl-imidazole 48-2-ethyl-imidazole 49-benzimidazole

Imidazole ring 50-59-imidazole 60-68-2-methyl-imidazole 69-77-2-ethyl-imidazole 78-87-benzimidazole<smiles>[R]C[N+]1([R])C=[N+]([Y])C=C1C(c1ccccc1)c1cc2ccccc2o1</smiles>

Compounds $50,60,69,78$ $51,61,70,79$ $52,62,71,80$

53,81

$54,63,72,82$

$55,64,73,83$

$56,65,74,84$

$57,66,75,85$

$58,67,76,86$

$59,68,77,87$

SCHEMe 11<smiles>[R][R15]CC</smiles>

SCHeme 12<smiles>[R1]C=CC(O)Cn1cc([N+](=O)[O-])nc1[R]</smiles>

$89 a-h$<smiles>[R][X]c1ccc(C(O)Cn2cc([N+](=O)[O-])nc2OC)cc1</smiles>

$90 a-h$

$$
\mathrm{R}_{1}
$$

90a 2,4-Dichloro

90b 2,4-Difluoro

90c 4-F

90d 4-Cl

90e $4-\mathrm{NO}_{2}$

90f 4-Phenyl

$90 \mathrm{~g} \mathrm{H}$

90h 2,4-Dimethyl

displayed excellent activity particularly against $P$. chrysogenum, almost equivalent to the standard drug Ketoconazole. Amongst the tested compounds, chlorosubstituted imidazolyl cinnamamide $16 \mathrm{c}$ showed strong antibacterial activity against B. subtilis [8] (see Scheme 7).

\section{Anticancer Activities}

Yang et al. synthesised series of novel hybrid compounds between 2-phenylbenzofuran and imidazole have been prepared and results suggest that substitution of the imidazolyl3-position with a naphthylacyl or bromophenacyl group, was vital for modulating cytotoxic activity. Compound 21 was the most active compound which displayed similar cytotoxic activity in vitro compared with DDP. Compared with alkyl substituent imidazolium salt derivatives 20-23, hybrid compounds 24-30 with phenacyl substituent at position-3 of imidazole ring exhibited higher cytotoxic activity. Compounds 28, 29, and 30 displayed similar cytotoxic activity in vitro compared with DDP [9] (see Scheme 8).

Alkahtani et al. synthesized and evaluated benzo[d] imidazole derivatives as potential anticancer agents. 5,6Dichloro-1-cyclopentyl-1H-benzo[d]imidazoles 31, 32, and 33 possessed potent antiproliferative activity in cancer cell lines [10] (see Scheme 9).

Ozkay et al. synthesized 2-substituted-N-[4-(1-methyl4,5-diphenyl-1H-imidazole-2-yl)phenyl] acetamide derivatives and evaluated anticancer activity. The 35, 38, 39, 40, and 41 are the most cytotoxic compounds in the series. Cytotoxicity of the 43 was lower than that of the 34 . The compounds 36, 37, and 42 showed approximate cytotoxic activity to the 34 . Compounds 44 and 45 revealed greater cytotoxic activity than the 34 . However, when concentrations were raised, cytotoxic activity of the 34 increased significantly 


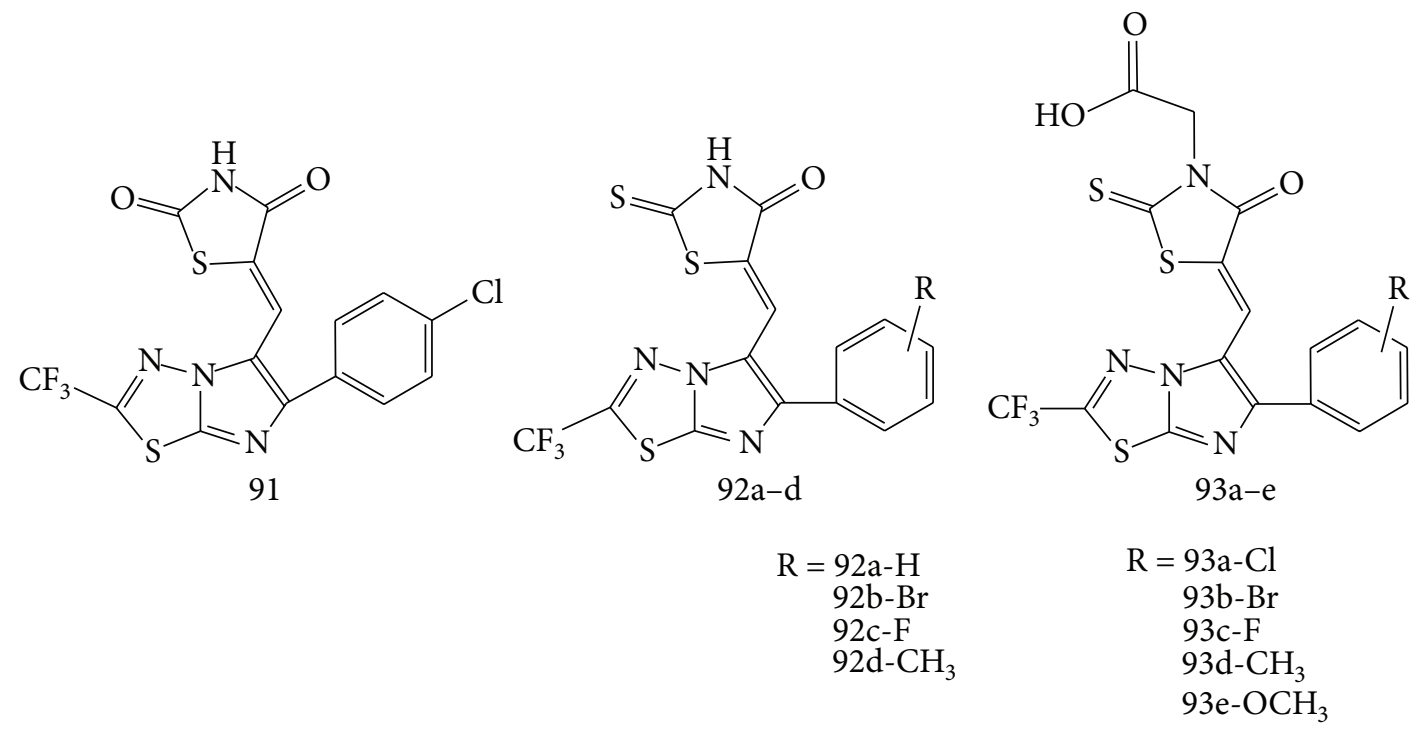

SCHEME 14<smiles>[Y17]NC(=O)C1=C(C)NC(C)=C(C(=O)N[Al])C1c1cnc(SC)n1[X]c1ccccc1</smiles>

\begin{tabular}{lll} 
& \multicolumn{1}{c}{ Ar } & $\mathrm{X}$ \\
94a & 2-Chlorophenyl & $\mathrm{NH}$ \\
94b & 3-Chlorophenyl & $\mathrm{NH}$ \\
94c & 4-Chlorophenyl & $\mathrm{NH}$ \\
94d & 4-Bromophenyl & $\mathrm{NH}$ \\
94e & 2-Pyridyl & $\mathrm{NH}$ \\
94f & 3-Pyridyl & $\mathrm{NH}$ \\
94g & 2-Chlorophenyl & $\mathrm{NH}$ \\
94h & 3-Chlorophenyl & $\mathrm{NH}$ \\
94i & 4-Chlorophenyl & $\mathrm{NH}$ \\
94j & 3-Pyridyl & $\mathrm{NH}$
\end{tabular}

SCHEME 15<smiles>[R]c1ccc(C2=NN([R1])C(n3ccnc3)C2c2ccc([R])cc2)cc1</smiles>

$\quad \mathrm{R}$
$95 \mathrm{a}-\mathrm{H}$
$95 \mathrm{~b}-\mathrm{Br}$
$95 \mathrm{c}-\mathrm{Cl}$
$95 \mathrm{~d}-2,4-(\mathrm{Cl})_{2}$
$95 \mathrm{e}-\mathrm{CH}_{3}$
$95 \mathrm{f}-\mathrm{H}$
$95 \mathrm{~g}-\mathrm{Br}$
$95 \mathrm{~h}-\mathrm{Cl}$
$95 \mathrm{i}-2,4-(\mathrm{Cl})_{2}$
$95 \mathrm{j}-\mathrm{CH}_{3}$

$\mathrm{R}_{1}$

$\mathrm{H} \quad 95 \mathrm{k}$

$\mathrm{H} \quad 951-\mathrm{Br}$

$\mathrm{H} \quad 95 \mathrm{~m}-\mathrm{Cl}$

$\mathrm{H}$

$95 \mathrm{n}-2,4-(\mathrm{Cl})$

$\mathrm{H} \quad 95 \mathrm{o}-\mathrm{CH}_{3}$

$\mathrm{CH}_{3} \quad 95 \mathrm{p}-\mathrm{H}$

$\mathrm{CH}_{3} \quad 95 \mathrm{q}-\mathrm{Br}$

$\mathrm{CH}_{3} \quad 95 \mathrm{r}-\mathrm{Cl}$

$\mathrm{X}$

$\mathrm{CH}_{3} \quad 95 \mathrm{~s}-2,4-(\mathrm{Cl})_{2}$

$\mathrm{CH}_{3} \quad 95 \mathrm{t}-\mathrm{CH}_{3}$

SCHEMe 16

$\mathrm{R}$<smiles>[R]CCCCc1cn2nc(-c3nccn3C)sc2n1</smiles>

96a-f 96a 3-Nitro

$96 \mathrm{~b}$ 4-Bromo

96c 4-Chloro

96d 4-Fluoro

96e 4-Nitro

96f 2,4-Dichloro 
<smiles>[R]n1ccnc1[2H]</smiles>

97-99<smiles>[R1]NC(=O)CN1CC=NC1[R]</smiles>

100-103
97: $\mathrm{R}=\mathrm{H}, \mathrm{R}_{1}=3$,4-dichlorobenzyl 98: $\mathrm{R}=\mathrm{H}, \mathrm{R}_{1}=\mathrm{CH}_{2} \mathrm{COOEt}$ 99: $\mathrm{R}=\mathrm{H}, \mathrm{R}_{1}=\mathrm{CH}_{2} \mathrm{CH}_{2} \mathrm{COOEt}$
100: $\mathrm{R}=\mathrm{H}, \mathrm{R}_{1}=n$-butyl

101: $\mathrm{R}=\mathrm{H}, \mathrm{R}_{1}=n$-hexyl

102: $\mathrm{R}=\mathrm{H}, \mathrm{R}_{1}=n$-heptyl

103: $\mathrm{R}=\mathrm{H}, \mathrm{R}_{1}=$ benzyl<smiles>[R]c1nccn1CCO</smiles>

104<smiles>c1ccc(CNCCn2ccnc2)cc1</smiles>

105<smiles>c1ccc2[nH]cnc2c1</smiles>

106<smiles>[R]c1ccc(Cn2cnc3ccccc32)cc1[R]</smiles>

107-108

107: $\mathrm{R}=\mathrm{R}_{1}=\mathrm{H}$ 108: $\mathrm{R}=\mathrm{R}_{1}=\mathrm{Cl}$<smiles>[R]c1nccn1COCn1c([R])cnc1[R]</smiles>

109-112

109: $n=1, \mathrm{R}=\mathrm{R}_{1}=\mathrm{H}$

110: $n=3, \mathrm{R}=\mathrm{R}_{1}=\mathrm{H}$

111: $n=1, \mathrm{R}=$ propyl, $\mathrm{R}_{1}=\mathrm{H}$

112: $n=1, \mathrm{R}=$ propyl, $\mathrm{R}_{1}=$ allyl<smiles>[R]c1nccn1C(CC(=O)OCC)[C@@H]1O[C@@H]2O[Z10](C)(C)O[C@@H]2[C@H]1[R20]</smiles>

113-116

113: $\mathrm{R}=\mathrm{CH}_{3} ; \mathrm{R}_{1}=\mathrm{H}$,

114: $\mathrm{R}=\mathrm{CH}_{2} \mathrm{Ph} ; \mathrm{R}_{1}=\mathrm{H}$,

115: $\mathrm{R}=\mathrm{CH}_{3} ; \mathrm{R}_{1}=$ propyl,

116: $\mathrm{R}=\mathrm{CH}_{2} \mathrm{Ph} ; \mathrm{R}_{1}=$ propyl

SCHEME 18<smiles>[R]N(C#N)C(=O)c1ncn([R1])c1C</smiles>
$\mathrm{R}_{1}$
$\mathrm{R}_{2}$
$117 \mathrm{a}-\mathrm{p}$

$117 \mathrm{a}-\mathrm{C}_{6} \mathrm{H}_{5} \mathrm{CH}_{2}$

$117 \mathrm{~b}-2-\mathrm{F}_{-} \mathrm{C}_{6} \mathrm{H}_{5} \mathrm{CH}_{2}$

$117 \mathrm{c}-2-\mathrm{F}_{-} \mathrm{C}_{6} \mathrm{H}_{5} \mathrm{CH}_{2}$

$117 \mathrm{~d}-2-\mathrm{F}_{-} \mathrm{C}_{6} \mathrm{H}_{5} \mathrm{CH}_{2}$

$117 \mathrm{e}-4-\mathrm{F}-\mathrm{C}_{6} \mathrm{H}_{5} \mathrm{CH}_{2}$

$117 \mathrm{f}-\mathrm{C}_{6} \mathrm{H}_{5} \mathrm{CH}_{2} \mathrm{CH}_{2} \mathrm{CH}_{2}$

$117 \mathrm{~g}-2-\mathrm{Cl}-\mathrm{C}_{6} \mathrm{H}_{5} \mathrm{CH}_{2}$

$117 \mathrm{~h}-2,4-\mathrm{F} 2-\mathrm{C}_{6} \mathrm{H}_{5} \mathrm{CH}_{2}$
$\mathrm{C}_{6} \mathrm{H}_{5} \mathrm{CH}_{2}$ $\mathrm{CH}_{3} \mathrm{CH}_{2} \mathrm{CH}_{2} \mathrm{CH}_{2}$ 4- $\mathrm{CH}_{3}-\mathrm{C}_{6} \mathrm{H}_{5} \mathrm{CH}_{2}$ $3-\mathrm{NO}_{2}-\mathrm{C}_{6} \mathrm{H}_{5} \mathrm{CH}_{2}$ $4-\mathrm{CH}_{3}-\mathrm{C}_{6} \mathrm{H}_{5} \mathrm{CH}_{2}$ $4-\mathrm{CH}_{3}-\mathrm{C}_{6} \mathrm{H}_{5} \mathrm{CH}_{2}$ $4-\mathrm{CH}_{3}-\mathrm{C}_{6} \mathrm{H}_{5} \mathrm{CH}_{2}$ $4-\mathrm{F}-\mathrm{C}_{6} \mathrm{H}_{5} \mathrm{CH}_{2}$
$117 \mathrm{i}-2-\mathrm{COOMe}-\mathrm{C}_{6} \mathrm{H}_{5} \mathrm{CH}_{2}$

$117 \mathrm{j}-4-\mathrm{F}-\mathrm{C}_{6} \mathrm{H}_{5} \mathrm{CH}_{2}$

$117 \mathrm{k}-4-\mathrm{CH}_{3}-\mathrm{C}_{6} \mathrm{H}_{5} \mathrm{CH}_{2}$

$117 \mathrm{l}-\mathrm{C}_{6} \mathrm{H}_{5} \mathrm{CH}_{2}$

$117 \mathrm{~m}-\mathrm{CH}_{3} \mathrm{CH}_{2} \mathrm{CH}_{2} \mathrm{CH}_{2}$

$117 \mathrm{n}-\mathrm{C}_{6} \mathrm{H}_{5} \mathrm{CH}_{2}$

$117 \mathrm{o}-\mathrm{C}_{6} \mathrm{H}_{5} \mathrm{CH}_{2}$

$117 \mathrm{p}-4-\mathrm{F}-\mathrm{C}_{6} \mathrm{H}_{5} \mathrm{CH}_{2}$
$4-\mathrm{F}-\mathrm{C}_{6} \mathrm{H}_{5} \mathrm{CH}_{2}$ $4-\mathrm{F}-\mathrm{C}_{6} \mathrm{H}_{5} \mathrm{CH}_{2}$ $4-\mathrm{CH}_{3}-\mathrm{C}_{6} \mathrm{H}_{5} \mathrm{CH}_{2}$ $4-\mathrm{F}-\mathrm{C}_{6} \mathrm{H}_{5} \mathrm{CH}_{2}$ $\mathrm{CH}_{3} \mathrm{CH}_{2} \mathrm{CH}_{2} \mathrm{CH}_{2}$ $\mathrm{CH}_{3} \mathrm{CH}_{2} \mathrm{CH}_{2} \mathrm{CH}_{2}$ 4- $\mathrm{CH}_{3}-\mathrm{C}_{6} \mathrm{H}_{5} \mathrm{CH}_{2}$ $3-\mathrm{NO}_{2}-\mathrm{C}_{6} \mathrm{H}_{5} \mathrm{CH}_{2}$ 
<smiles>[Y13]c1ccc(C(O)Cn2ccnc2)cc1</smiles><smiles>[X]c1ccc(C(Cn2ccnc2)OC(=O)COc2ccccc2)cc1</smiles>

$122 \mathrm{~b}-\mathrm{c}$<smiles>[Y]c1ccc(C(Cn2ccnc2)OC(=O)c2ccc([R3])c([R2])c2[R])cc1</smiles>

$119 a-d, 120 a, 121 a-c$
$\mathrm{X}=\mathrm{H}, \mathrm{F}, \mathrm{Cl}$

$$
\begin{aligned}
& 119 \mathrm{a}-\mathrm{R}_{1}=\mathrm{R}_{3}=\mathrm{H}, \mathrm{R}_{2}=\mathrm{CF}_{3}, \mathrm{X}=\mathrm{H} \\
& 119 \mathrm{~b}-\mathrm{R}_{1}=\mathrm{R}_{3}=\mathrm{H}, \mathrm{R}_{2}=\mathrm{CF}_{3}, \mathrm{X}=\mathrm{F} \\
& 119 \mathrm{c}-\mathrm{R}_{1}=\mathrm{R}_{3}=\mathrm{H}, \mathrm{R}_{2}=\mathrm{CF}_{3}, \mathrm{X}=\mathrm{Cl} \\
& 119 \mathrm{~d}-\mathrm{R}_{1}=\mathrm{R}_{3}=\mathrm{H}, \mathrm{R}_{2}=\mathrm{CF}_{3}, \mathrm{X}=\mathrm{CF}_{3}
\end{aligned}
$$<smiles>[Y16]CCCCC(C)c1ccc(NC(=O)OC(Cn2ccnc2)c2ccc([Y4])cc2)cc1</smiles>

Scheme 20<smiles>[X]c1ccc(C(Cn2ccnc2)OC(=O)Nc2cc(Cl)nc(Cl)c2)cc1</smiles><smiles>[R]c1ccc(C2C([R])C(=O)C([R])C(c3ccc([R])c([R])c3)N2C(=O)Cn2ccnc2)cc1[R]</smiles>

Scheme 21<smiles>O=C(I)NN1C(=O)/C(=C/c2cc3ccccc3nc2Cl)N=C1c1ccccc1</smiles>

$$
\begin{array}{ll} 
& -\mathrm{R} \\
135 \mathrm{a} & -\mathrm{C}_{6} \mathrm{H}_{5} \\
135 \mathrm{~b} & \mathrm{C}_{6} \mathrm{H}_{5}-\mathrm{CH}_{2} \\
135 \mathrm{c} & -3-\mathrm{Cl}-\mathrm{C}_{6} \mathrm{H}_{4} \\
135 \mathrm{~d} & -4-\mathrm{Cl}-\mathrm{C}_{6} \mathrm{H}_{4} \\
135 \mathrm{e} & -2,5-(\mathrm{Cl})_{2}-\mathrm{C}_{6} \mathrm{H}_{3} \\
135 \mathrm{f} & -4-\mathrm{F}-\mathrm{C}_{6} \mathrm{H}_{4} \\
135 \mathrm{~g} & -3-\mathrm{NO}_{2}-\mathrm{C}_{6} \mathrm{H}_{4}
\end{array}
$$

$135 \mathrm{~h}-4-\mathrm{NO}_{2}-\mathrm{C}_{6} \mathrm{H}_{4}$

$135 \mathrm{i}-2-\mathrm{OH}-\mathrm{C}_{6} \mathrm{H}_{4}$

$135 \mathrm{j}-3-\mathrm{OH}-\mathrm{C}_{6} \mathrm{H}_{4}$

$135 \mathrm{k}-2-\mathrm{OH}-4-\mathrm{Cl}-\mathrm{C}_{6} \mathrm{H}_{3}$

$135 \mathrm{l}-\mathrm{C}_{5} \mathrm{H}_{4} \mathrm{~N}$ 
<smiles>COC(=O)/C=C1/C(=O)NC(=S)N1/N=C/c1c[nH]nc1[Al]</smiles>

136a 2,4-Dichlorophenyl 136b 2,5-Dichlorothiophene $136 \mathrm{c}$ $136 \mathrm{~d}$ $4-\mathrm{SCH}_{3}-\mathrm{C}_{6} \mathrm{H}_{4}$ 4- $\mathrm{CH}_{3}-\mathrm{C}_{6} \mathrm{H}_{4}$
$137 \mathrm{a}$

$137 \mathrm{~b}$

$137 \mathrm{c}$

$137 \mathrm{~d}$

$137 \mathrm{e}$

$137 \mathrm{f}$

$137 \mathrm{~g}$

$137 \mathrm{~h}$

$137 \mathrm{i}$

$137 \mathrm{j}$<smiles></smiles>

$$
\begin{array}{cc}
\text { 4- } \mathrm{SCH}_{3}-\mathrm{C}_{6} \mathrm{H}_{4} & \mathrm{H} \\
\text { 2,4-Dichlorophenyl } & \mathrm{H} \\
\text { Biphenyl } & \mathrm{H} \\
\text { 4- } \mathrm{CH}_{3}-\mathrm{C}_{6} \mathrm{H}_{4} & \mathrm{H} \\
\text { 2,5-Dichlorothiophene } & \mathrm{H} \\
\text { 4- } \mathrm{SCH}_{3}-\mathrm{C}_{6} \mathrm{H}_{4} & \mathrm{Br} \\
\text { 2,4-Dichlorophenyl } & \mathrm{Br} \\
\text { Biphenyl } & \mathrm{Br} \\
\text { 4- } \mathrm{CH}_{3}-\mathrm{C}_{6} \mathrm{H}_{4} & \mathrm{Br} \\
\text { 2,5-Dichlorothiophene } & \mathrm{Br}
\end{array}
$$

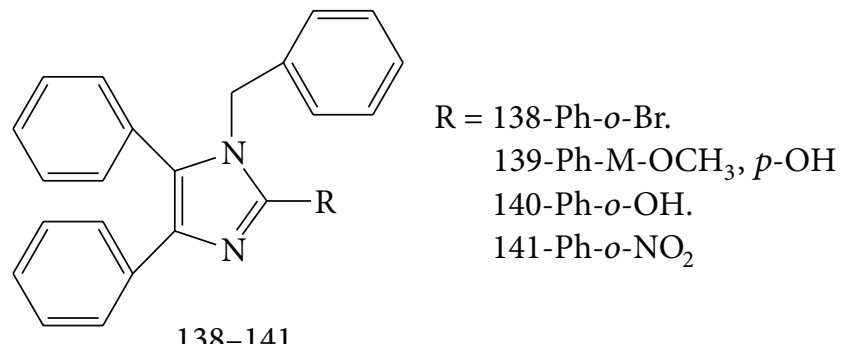

Scheme 24

and seemed as higher than those of the 44 and 45 [11] (see Scheme 10).

Wang et al. evaluated the cytotoxic potential of all newly synthesized hybrid compounds. 2-Benzylbenzofurane imidazole hybrids 47-49 lacked activities against all tumor cell lines. In terms of the imidazole ring (imidazole, 2methyl-imidazole or 2-ethyl-imidazole, and benzimidazole), imidazolium salt hybrids 50-59 with imidazole ring displayed weak cytotoxic activities. Only compounds 55 and 58 showed medium cytotoxic activities, and compound 59 with a naphthylacyl substituent at position-3 of the imidazole ring displayed higher cytotoxic activity in vitro. Imidazolium salt hybrids 60-68 with 2-methyl-imidazole ring and 6977 with 2-ethyl-imidazole ring exhibited medium cytotoxic activities. However, imidazolium salt hybrids $78-87$ with benzimidazole ring exhibited powerful cytotoxic activities. Among them, compounds 86 and 87 showed potent cytotoxic activities [12] (see Scheme 11).

\section{Antitubercular Activity}

Lu et al. synthesised a series of substituted 4-(2,6-dichlorobenzyloxy)phenyl thiazole, oxazole and imidazole derivatives. The derivatives were screened for in vitro antitubercular activities against Mycobacterium tuberculosis $\mathrm{H} 37 \mathrm{Rv}$ [13] (see Scheme 12).

Lee et al. synthesised monocyclic nitroimidazole derivatives, and the antitubercular activity of the synthesized compounds against $\mathrm{Mtb} \mathrm{H} 37 \mathrm{Rv}$ was determined by the microdilution Alamar blue assay. Compounds 89a, 89b, and $89 \mathrm{~d}$ were moderately active. In case of $90 \mathrm{a}, 90 \mathrm{~d}, 90 \mathrm{e}$, and $90 \mathrm{~g}$, the activity was increased 4 -fold, compared with $89 \mathrm{~b}, 89 \mathrm{e}, 89 \mathrm{f}$, and $89 \mathrm{~g}$, respectively. While $90 \mathrm{c}$ and $90 \mathrm{~h}$ were 8 -fold more active than $89 \mathrm{~d}$ and $89 \mathrm{~h}$, respectively, $90 \mathrm{~b}$ was 16 -fold more active than 90c [14] (see Scheme 13).

Alegaon et al. synthesized imidazo[2,1-b][1,3,4]thiadiazole derivatives, and the antitubercular activities have been assessed against $M$. tuberculosis H37Rv (ATCC 27294) and found that compounds (91, 92a, 92b, 92c, 92d, 93a, 93b, 93c, 93d, and 93e) are active against M. tuberculosis [15] (see Scheme 14).

According to Fassihi et al. a series of 4-substituted imidazolyl-2,6-dimethyl-N3,N5-bisaryl-1,4-dihydropyridine-3,5dicarboxamides $(94 \mathrm{a}-\mathrm{j})$ were prepared and tested in vitro against $M$. tuberculosis H37RV strain ATCC 27294 which is susceptible to rifampicin and isoniazid [16] (see Scheme 15).

According to Zampieri et al. a series of 1-(3,5-diaryl-4,5dihydro-1H-pyrazol-4-yl)-1H-imidazole and 1-[(1-aralkyl)3,5-diaryl-4,5-dihydro-1H-pyrazol-4-yl]-1H-imidazole derivatives were synthesized and evaluated for antimycobacterial activities. Compounds $95 \mathrm{a}-\mathrm{t}$ were tested against a strain of 


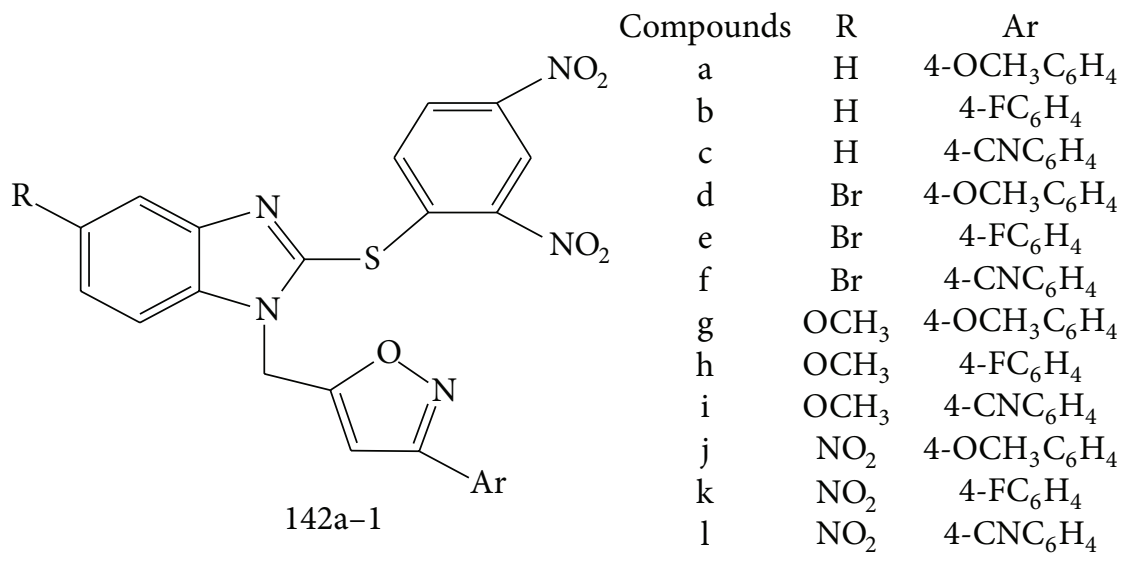

SCHEME 25

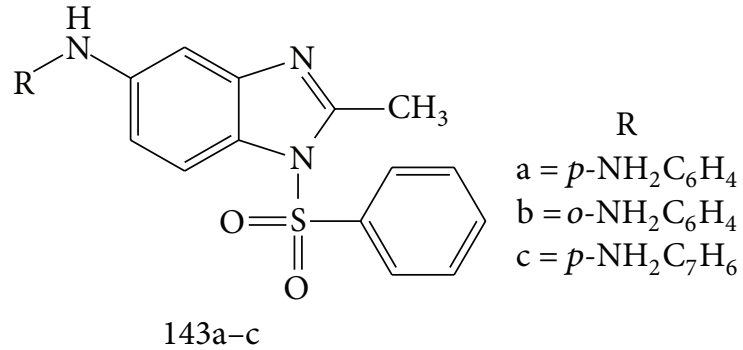

Scheme 26

M. tuberculosis $\mathrm{H} 37 \mathrm{Rv}$ and showed a good antimycobacterial activity [17] (see Scheme 16).

Imidazo[2,1-b] $[1,3,4]$ thiadiazole derivatives were synthesised by Patel et al. and evaluated for in vitro antitubercular activity against $M$. tuberculosis strain H37Rv. Among the imidazo[2,1-b] $[1,3,4]$ thiadiazole series the compounds 96a$\mathrm{f}$ exhibited significant antitubercular activities but not as good as that of the nitro phenyl substituent 96e [18] (see Scheme 17).

Pandey et al. synthesized and screened a series of imidazole-based compounds (97-116) for their antitubercular efficacy against $M$. tuberculosis [19] (see Scheme 18).

\section{Antifungal Activities}

Yang et al. synthesized various $\mathrm{N}$-cyano-1H-imidazole-4carboxamides derivatives, and the fungicidal activities were screened against six kinds of fungi, Fusarium oxysporum, Rhizoctonia solani, Botrytis cinerea Pers, Gibberella zeae, Dothiorella gregaria, and Colletotrichum gossypii, at a concentration of $50 \mathrm{lg} / \mathrm{mL}$. The newly synthesized compounds have good antifungal activity selectively against Rhizoctonia solani among the six fungi tested. Particularly, compound $117 \mathrm{~h}$ was identified as the most promising candidate with an EC50 of $2.63 \mathrm{lg} / \mathrm{mL}$ against $R$. Solani [20] (see Scheme 19).

The in vitro antifungal activity of imidazole derivatives $118 \mathrm{a}-\mathrm{c}, 119 \mathrm{a}-\mathrm{d}, 120 \mathrm{a}, 121 \mathrm{a}-\mathrm{c}, 122 \mathrm{~b}-\mathrm{c}, 123 \mathrm{a}-\mathrm{c}$, and 124c was evaluated by Vita et al. against four strains of $C$. albicans and seven strains of nonalbicans Candida species [21] (see Scheme 20).

Ramachandran et al. synthesised various imidazole derivatives and evaluated fungicidal activity against $A$. niger, C. neoformans, Rhizopus sp., C. albicans, and A. flavus [7] (see Scheme 21).

Desai et al. synthesised N-(4-((2-chloroquinolin-3yl)methylene)-5-oxo-2-phenyl-4,5-dihydro-1H-imidazol-1yl)(aryl) amides (135a-l). The compounds were tested for antifungal activity in six sets against C. albicans, Aspergillus niger and A. clavatus at various concentrations. Among these compounds $135 \mathrm{c}, 135 \mathrm{~d}, 135 \mathrm{f}, 135 \mathrm{~h}$, and $135 \mathrm{j}$ showed significant potency against different microbial strains [22] (see Scheme 22).

Vijesh et al. synthesized and screened compounds 136a$\mathrm{d}$ and 137a-j for their antifungal activity against Aspergillus flavus, Aspergillus niger, Candida albicans, Microsporum gypseum, and Trichophyton rubrum. Among the tested compounds, the compound $136 \mathrm{c}$ has emerged as active against T. rubrum compared with standard, fluconazole [4] (see Scheme 23).

\section{Analgesic Activity}

Ucucu et al. reported the synthesis of some 1-benzyl2-substituted-4,5-diphenyl-1H-imidazole derivatives. Swiss albino mice were used to carry out analgesic activity of both sexes weighing $23-36 \mathrm{~g}$. All derivatives show poor response; only compounds 138 and 139 exhibited a moderate activity, and compounds 140 and 141 ranged not far from morphine [23] (see Scheme 24).

According to Kankala et al. synthesis of isoxazolemercaptobenzimidazole hybrids and the analgesic activity of the synthesized compounds (142a-1) was assessed by hot plate method. Almost all the compounds have shown very potent analgesic activity when compared with standard drug pentazocine. Amongst all the compounds, 142e and $142 \mathrm{f}$ with potent analgesic activity, the compounds $142 \mathrm{k}$ and 1421 have shown moderate activity and were found to be more potent than the standard pentazocine. The remaining 


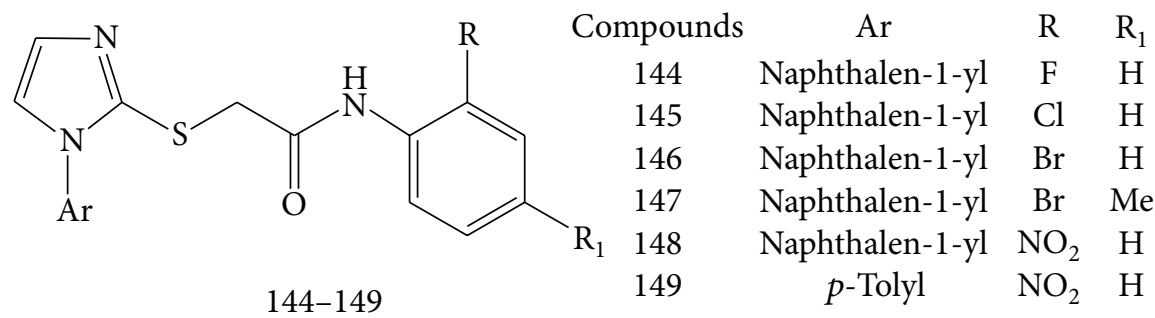

SCHEME 27

compounds $142 \mathrm{a}-\mathrm{d}$ and $142 \mathrm{~g}-\mathrm{j}$ had shown poor activity [24] (see Scheme 25).

A series of novel 5-substituted-1-(phenylsulfonyl)-2methylbenzimidazole derivatives have been synthesized and evaluated for analgesic activity. Derivatives 143a-c exhibited moderate to good analgesic activity [25] (see Scheme 26).

\section{Anti-HIV Evaluation}

A series of 2-(1-aryl-1H-imidazol-2-ylthio)acetamide [imidazole thioacetanilide (ITA)] derivatives were synthesized and evaluated as potent inhibitors of human immunodeficiency virus type-1 (HIV-1). All of the newly synthesized imidazole thioacetanilides were first evaluated for their anti-HIV activity. The most potent HIV-1 inhibitors were 148 and 145. Other compounds, 146, 147, 149, and 144, also showed higher antiHIV-1 potency [26] (see Scheme 27).

\section{Conclusion}

The above study about various imidazole derivatives is the significant class of heterocyclic compounds, showed promising results in most of the pharmacological activities, and also has fascinating results including antibacterial, anticancer, antitubercular, antifungal, analgesic, and anti-HIV activities. It has been noticed so far that modifications on imidazole nucleus displayed promising biological activities. It will be interesting to observe that in the future many new pharmacological profiles will be added to it as it is still unrevealed and can be taken as a lead for future development to get safer and more effective compounds.

\section{Conflicts of Interests}

Authors declare that there is no conflict of interests regarding the publication of this article.

\section{References}

[1] A. Kleeman, J. Engel, B. Kutscher, and D. Reichert, Pharmaceutical Substances: Syntheses, Patents, Applications of the Most Relevant APIs, Thieme Medical, New York, NY, USA, 3rd edition, 1999.

[2] H. Debus, "Ueber die Einwirkung des Ammoniaks auf Glyoxal," Annalen der Chemie und Pharmacie, vol. 107, no. 2, pp. 199-208, 1858.
[3] H. Singh and V. K. Kapoor, Medicinal and Pharmaceutical Chemistry, vol. 2, Vallabh Prakashan, Delhi, India, 2008.

[4] A. M. Vijesh, A. M. Isloor, S. Telkar, S. K. Peethambar, S. Rai, and N. Isloor, "Synthesis, characterization and antimicrobial studies of some new pyrazole incorporated imidazole derivatives," European Journal of Medicinal Chemistry, vol. 46, no. 8, pp. 3531-3536, 2011.

[5] X. Lu, X. Liu, B. Wan et al., "Synthesis and evaluation of anti-tubercular and antibacterial activities of new 4-(2,6dichlorobenzyloxy)phenyl thiazole, oxazole and imidazole derivatives," European Journal of Medicinal Chemistry, vol. 49, pp. 164-171, 2012.

[6] A. K. Jain, V. Ravichandran, M. Sisodiya, and R. K. Agrawal, "Synthesis and antibacterial evaluation of 2-substituted-4,5diphenyl-N-alkyl imidazole derivatives," Asian Pacific Journal of Tropical Medicine, vol. 3, no. 6, pp. 471-474, 2010.

[7] R. Ramachandran, M. Rani, S. Senthan, Y. T. Jeong, and S. Kabilan, "Synthesis, spectral, crystal structure and in vitro antimicrobial evaluation of imidazole/benzotriazole substituted piperidin-4-one derivatives," European Journal of Medicinal Chemistry, vol. 46, no. 5, pp. 1926-1934, 2011.

[8] V. Padmavathi, C. P. Kumari, B. C. Venkatesh, and A. Padmaja, "Synthesis and antimicrobial activity of amido linked pyrrolyl and pyrazolyl-oxazoles, thiazoles and imidazoles," European Journal of Medicinal Chemistry, vol. 46, no. 11, pp. 5317-5326, 2011.

[9] X. Yang, W. Wan, X. Deng et al., "Design, synthesis and cytotoxic activities of novel hybrid compounds between 2phenylbenzofuran and imidazole," Bioorganic and Medicinal Chemistry Letters, vol. 22, no. 8, pp. 2726-2729, 2012.

[10] H. M. Alkahtani, A. Y. Abbas, and S. Wang, "Synthesis and biological evaluation of benzo[d]imidazole derivatives as potential anti-cancer agents," Bioorganic and Medicinal Chemistry Letters, vol. 22, no. 3, pp. 1317-1321, 2012.

[11] Y. Özkay, I. Işikdağ, Z. Incesu, and G. Akalin, "Synthesis of 2-substituted-N-[4-(1-methyl-4,5-diphenyl-1H-imidazole-2yl) phenyl] acetamide derivatives and evaluation of theiranticancer activity," European Journal of Medicinal Chemistry, vol. 45, no. 8, pp. 3320-3328, 2010.

[12] X. Wang, L. Liu, Y. Li et al., "Design, synthesis and biological evaluation of novel hybrid compounds of imidazole scaffoldbased 2-benzylbenzofuran as potent anticancer agents," European Journal of Medicinal Chemistry, vol. 62, pp. 111-121, 2013.

[13] X. Lu, X. Liu, B. Wan et al., "Synthesis and evaluation of anti-tubercular and antibacterial activities of new 4-(2,6dichlorobenzyloxy)phenyl thiazole, oxazole and imidazole derivatives," European Journal of Medicinal Chemistry, vol. 49, pp. 164-171, 2012.

[14] S. Lee, S. Kim, M. Yun et al., "Synthesis and antitubercular activity of monocyclic nitroimidazoles: insights from econazole," 
Bioorganic and Medicinal Chemistry Letters, vol. 21, no. 5, pp. 1515-1518, 2011.

[15] S. G. Alegaon, K. R. Alagawadi, P. V. Sonkusare, S. M. Chaudhary, D. H. Dadwe, and A. S. Shah, "Novel imidazo[2,1b] $[1,3,4]$ thiadiazole carrying rhodanine-3-acetic acid as potential antitubercular agents," Bioorganic and Medicinal Chemistry Letters, vol. 22, no. 5, pp. 1917-1921, 2012.

[16] A. Fassihi, Z. Azadpour, N. Delbari et al., "Synthesis and antitubercular activity of novel 4-substituted imidazolyl-2,6dimethyl-N3,N5-bisaryl-1,4-dihydropyridine-3,5-dicarboxamides," European Journal of Medicinal Chemistry, vol. 44, no. 8, pp. 3253-3258, 2009.

[17] D. Zampieri, M. G. Mamolo, E. Laurini, G. Scialino, E. Banfi, and L. Vio, "Antifungal and antimycobacterial activity of 1-(3,5-diaryl-4,5-dihydro-1H-pyrazol-4-yl)-1H-imidazole derivatives," Bioorganic and Medicinal Chemistry, vol. 16, no. 8, pp. 4516-4522, 2008.

[18] H. M. Patel, M. N. Noolvi, N. S. Sethi, A. K. Gadad, and S. S. Cameotra, "Synthesis and antimicrobial evaluation of novel 1,3,4-thiadiazole derivatives of 2-(4-formyl-2methoxyphenoxy) acetic acid," Arabian Journal of Chemistry, pp. 1-22, 2013.

[19] J. Pandey, V. K. Tiwari, S. S. Verma et al., "Synthesis and antitubercular screening of imidazole derivatives," European Journal of Medicinal Chemistry, vol. 44, no. 8, pp. 3350-3355, 2009.

[20] W. C. Yang, J. Li, J. Li, Q. Chen, and G. F. Yang, "Novel synthetic methods for N-cyano-1H-imidazole-4-carboxamides and their fungicidal activity," Bioorganic and Medicinal Chemistry Letters, vol. 22, no. 3, pp. 1455-1458, 2012.

[21] D. de Vita, L. Scipione, S. Tortorella et al., "Synthesis and antifungal activity of a new series of 2-(1H-imidazol-1-yl)1-phenylethanol derivatives," European Journal of Medicinal Chemistry, vol. 49, pp. 334-342, 2012.

[22] N. C. Desai, A. S. Maheta, K. M. Rajpara, V. V. Joshi, H. V. Vaghani, and H. M. Satodiya, "Green synthesis of novel quinoline based imidazole derivatives and evaluation of their antimicrobial activity," Journal of Saudi Chemical Society, 2011.

[23] Ü. Uçucu, N. G. Karaburun, and I. Işikdag, "Synthesis and analgesic activity of some 1-benzyl-2-substituted-4,5-diphenyl1H-imidazole derivatives," Farmaco, vol. 56, no. 4, pp. 285-290, 2001.

[24] S. Kankala, R. K. Kankala, G. Prasad et al., "Regioselective synthesis of isoxazole-mercaptobenzimidazole hybrids and their in vivo analgesic and anti-inflammatory activity studies," Bioorganic \& Medicinal Chemistry Letters, vol. 23, pp. 13061309, 2013.

[25] M. Gaba, D. Singh, S. Singh, V. Sharma, and P. Gaba, "Synthesis and pharmacological evaluation of novel 5-substituted1-(phenylsulfonyl)-2-methylbenzimidazole derivatives as antiinflammatory and analgesic agents," European Journal of Medicinal Chemistry, vol. 45, no. 6, pp. 2245-2249, 2010.

[26] P. Zhan, X. Liu, J. Zhu et al., "Synthesis and biological evaluation of imidazole thioacetanilides as novel non-nucleoside HIV1 reverse transcriptase inhibitors," Bioorganic and Medicinal Chemistry, vol. 17, no. 16, pp. 5775-5781, 2009. 

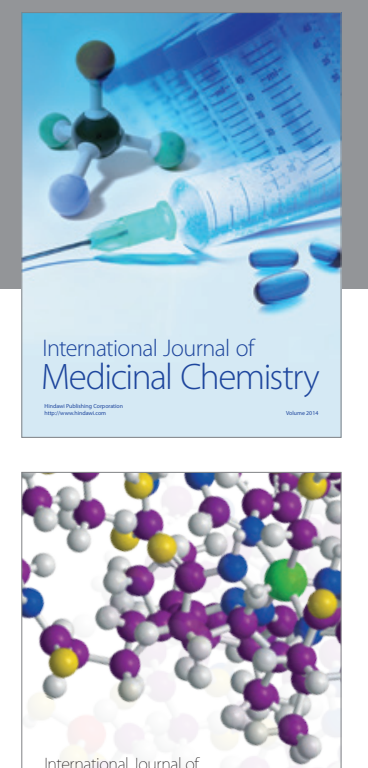

\section{Carbohydrate} Chemistry

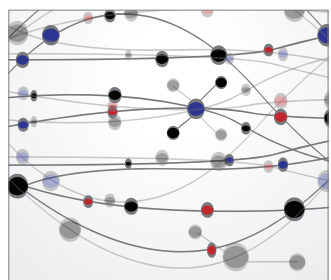

The Scientific World Journal
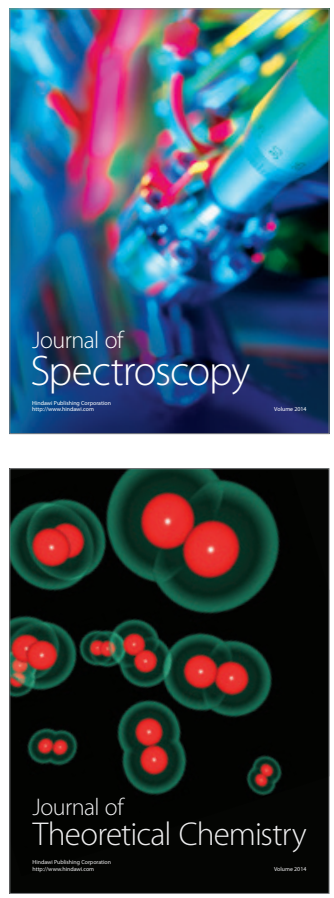
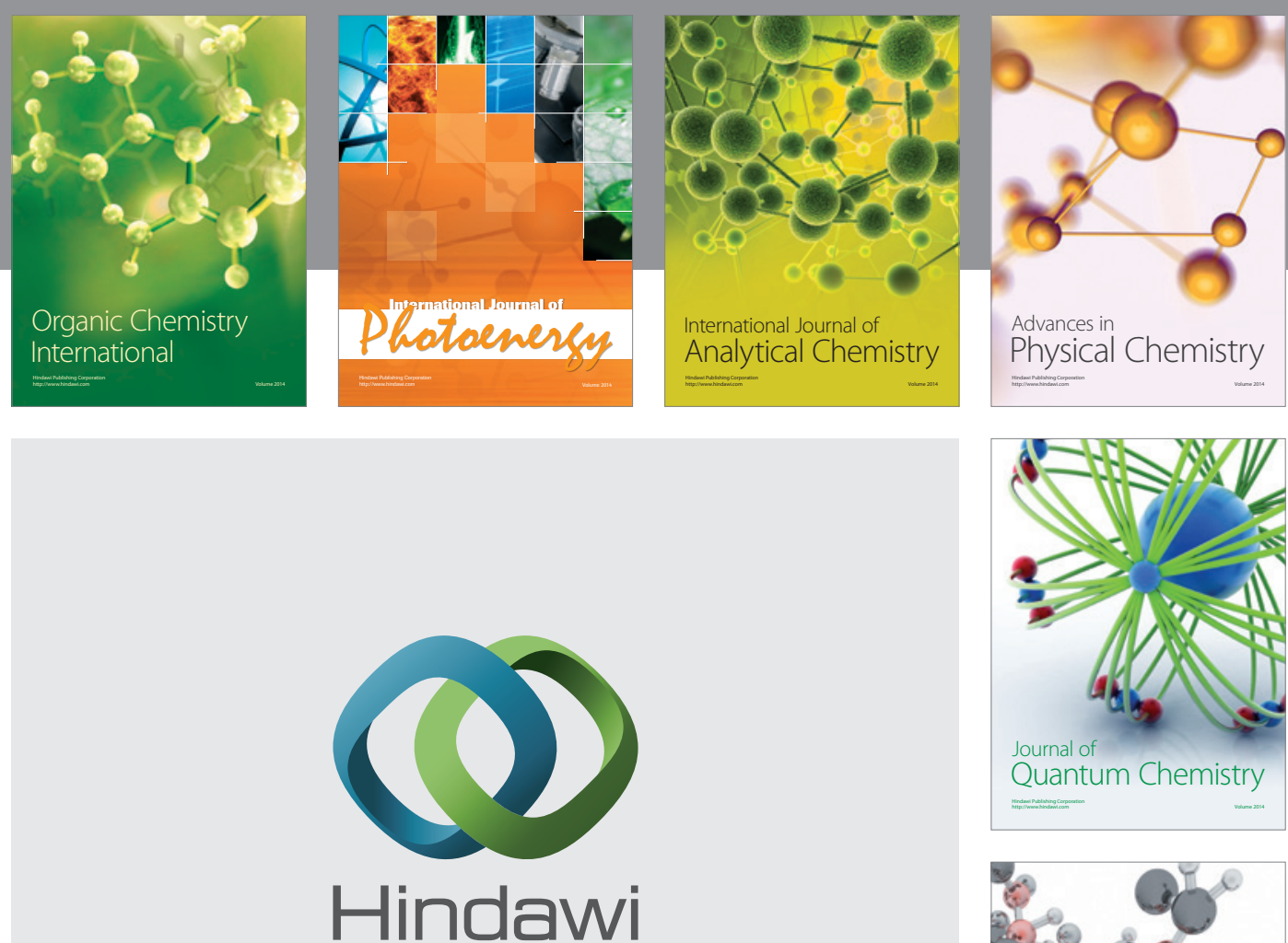

Submit your manuscripts at

http://www.hindawi.com

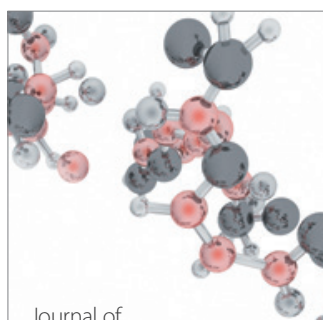

Analytical Methods

in Chemistry

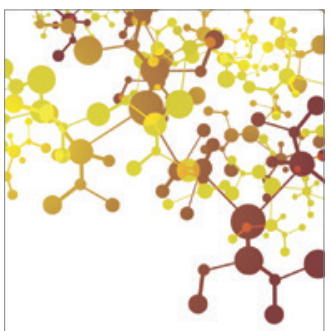

Journal of

Applied Chemistry

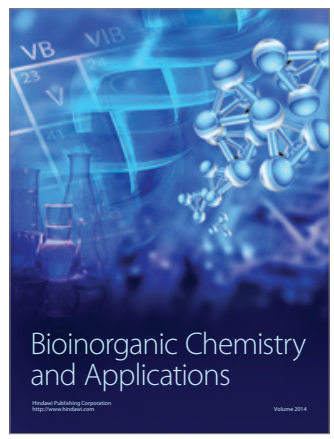

Inorganic Chemistry
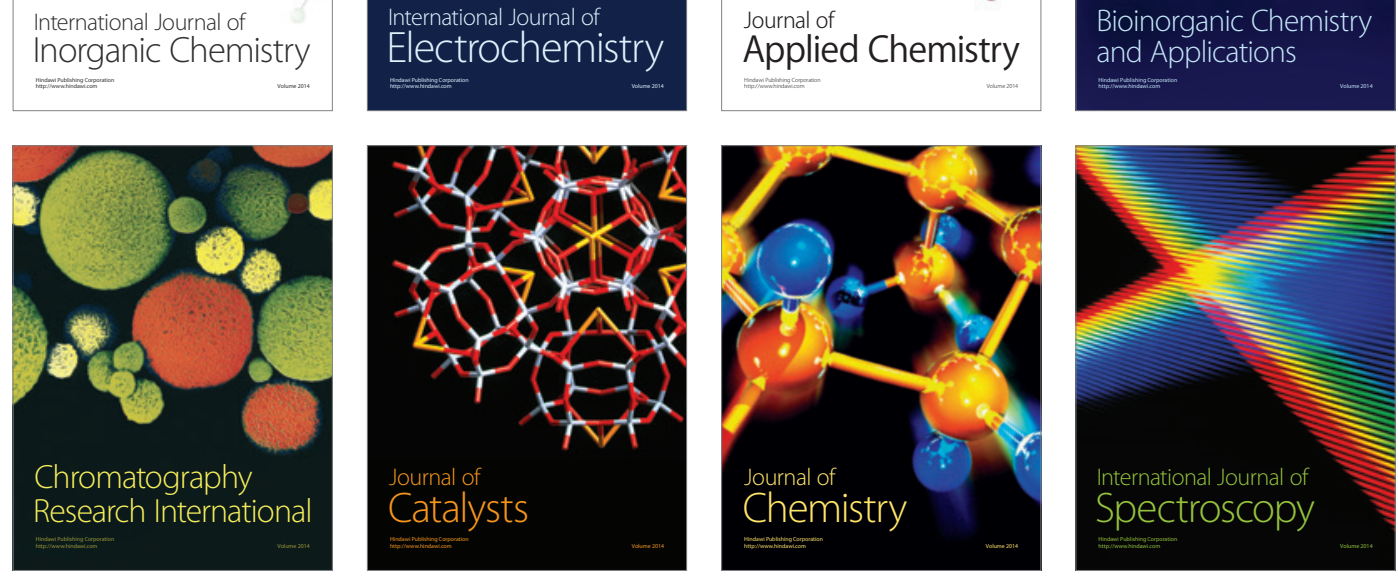